\title{
Analysis of Medium Access Control Protocols for Home Networks
}

\author{
Marcelo G. Rubinstein, Member, IEEE, Luís Henrique M. K. Costa, Member, IEEE, \\ Miguel Elias M. Campista, Student Member, IEEE, \\ Daniel de O. Cunha, Aurelio Amodei Jr., Pedro B. Velloso, and Otto Carlos M. B. Duarte
}

\begin{abstract}
Nowadays, there are many different home networking solutions: wired, wireless, and the so called "no new wires"; all compete for their market share. The most widely used metric to compare these technologies is the physical rate. Nevertheless, this metric does not reflect the peculiarities of each MAC protocol, which limit the bandwidth actually available to users. In this article, we analyze different home networking technologies taking the main features of their MAC protocols into account. We have chosen the saturation throughput as the basic metric and have provided analytical results. Then, through simulations, we have varied the number of nodes in the network to verify how each protocol deals with contention and to analyze their efficiency. Results show that collision-avoidance protocols have lower efficiency than collision-detection protocols. Nevertheless, there may be exceptions. HomePNA 3.0 has a relatively low efficiency because it uses the same basic rate as HomePNA 2.0, to keep compatibility. The same happens within a protocol family; IEEE 802.11g at $54 \mathrm{Mbps}$ is less efficient than IEEE 802.11b at 11 Mbps.
\end{abstract}

Index Terms-Home networks, medium access control, throughput analysis.

\section{INTRODUCTION}

$\mathbf{H}$ OME networks aim to interconnect home devices, such as computers, network devices, and household appliances, generally restricted to nodes separated by no more than $300 \mathrm{~m}$. These networks can be classified as wired, wireless, and "no new wires" [1]. Wired networks use specific cables, which are not available in most homes. Wireless networks use radio frequency and do not use cables. Phone or power lines, which are already deployed in the house, can be used to create no new wires networks. These networks do not require additional cabling and thus can be deployed at low costs.

Concerning wired networks, Ethernet [2] is the most widespread solution, but most homes do not have the infrastructure needed. The installation cost of new wires can be high. Fast Ethernet is presently being used where the required infrastructure is available, but Gigabit Ethernet may reach this niche as price goes down. On the other hand, wireless networks are now a huge success. The wireless technology has no contenders if mobility is considered, but presents problems related to performance, coverage, and quality of

This work was supported by CAPES, CNPq, FAPERJ, FINEP, and FUJB.

M. G. Rubinstein is with Universidade do Estado do Rio de Janeiro, Rio de Janeiro, RJ, Brazil.

L. H. M. K. Costa, M. E. M. Campista, A. Amodei Jr., and O. C. M. Duarte are with Universidade Federal do Rio de Janeiro, Rio de Janeiro, RJ, Brazil.

D. O. Cunha and P. B. Velloso are with the LIP6/CNRS Laboratory of UPMC Univ Paris, Paris, France. service guarantee, besides security. IEEE 802.11 [3] is the most widespread wireless LAN technology. IEEE 802.11b [4] operates in the $2.4 \mathrm{GHz}$ band and provides a maximum physical rate of $11 \mathrm{Mbps}$. IEEE 802.11a [5] supports physical rates of up to $54 \mathrm{Mbps}$ in the $5 \mathrm{GHz}$ band. The most recent specification is IEEE 802.11g [6], which can reach up to $54 \mathrm{Mbps}$ in the $2.4 \mathrm{GHz}$ band. Most IEEE 802.11 products are compliant with IEEE $802.11 \mathrm{~b}$ and IEEE $802.11 \mathrm{~g}$, and some support the three standards. Other wireless technologies such as, Bluetooth [7], ZigBee [8], and HiperLAN [9], are not evaluated in this paper. Bluetooth and ZigBee are only used in personal communications because of their small coverage and rate. On the other hand, HiperLAN has not reached commercial success.

In the last few years, no new wires technologies received special attention due to their ubiquity and low cost infrastructure. Home Phoneline Network Alliance (HomePNA) defined a standard for data transmission over home phonelines [10]. HomePNA 2.0 [11], [12], [13] supports physical data rates of up to 32 Mbps. HomePNA 3.0 can use two Medium Access Control (MAC) protocols: an asynchronous one (AMAC) and a synchronous one (SMAC). HomePNA 3.0 can reach up to $128 \mathrm{Mbps}$, with an optional extension to $240 \mathrm{Mbps}$. Home Powerline Network Alliance (HomePlug) defined a standard for data transmission over home powerlines [14]. HomePlug 1.0 supports physical data rates of 14 Mbps. A new standard called HomePlug AV is also being developed. Other powerline technologies, such as X10 and CEBus [15], are not considered in this paper because they are specific to home device control.

Home network applications range from distribution of information (audio, video, and data) to sharing Internet access. The main quality of service metric for many applications is bandwidth. As a consequence, to sell a technology, marketing is often based on the transmission rate at the physical layer. Nevertheless, the physical layer rate may not be the most appropriate parameter to be taken into account since the link layer necessarily limits the maximum throughput achievable. For shared medium, different MAC protocols have different efficiencies. Therefore, the maximum throughput provided by the MAC sub-layer of different home network technologies has to be considered when comparing those technologies.

Several researchers investigate the performance of home network MAC protocols. The saturation throughput of Ethernet has been extensively analyzed. Wang and Keshav [16] present performance results through simulation whereas Boggs et 
al. [17] perform measurements on an Ethernet network. Considering HomePNA, Chung et al. [18] and Kangude et al. [19] present mathematical analyses of the saturation throughput of HomePNA 2.0 and Kim et al. [20] perform a similar analysis for the HomePNA 3.0 AMAC. Jun et al. [21], Xiao et al. [22], Anastasi et al. [23], and Wijesinha et al. [24] analyze the theoretical saturation throughput of IEEE 802.11, 802.11b, 802.11a, and 802.11g. Doufexi et al. [25] present a throughput evaluation for 802.11a and 802.11g through simulation in different radio propagation conditions. Their results are specific to the scenario, which has one access point and other few nodes. Wijesinha et al. [24] present experimental results on a network of four nodes. HomePlug networks have also been evaluated. Lin et al. [1] and Jung et al. [26] present the theoretical saturation throughput of HomePlug 1.0. Lee et al. [27] analyze the throughput for HomePlug 1.0 through simulation on a network of only three nodes. Experimental results are presented by [1] and [27], but the authors only consider networks of a few nodes.

To the best of our knowledge, there is no work that makes a thorough comparison of medium access control techniques used by different home network technologies. Thus, the main objective of this paper is to analyze the peculiarities of these different techniques. We use mathematical analysis to evaluate the one-node maximum throughput of Ethernet, HomePNA 2.0 and 3.0 AMAC, IEEE 802.11b and g, and HomePlug 1.0. We verify our analyses by simulation. Then, we also evaluate the saturation throughput on scenarios with higher number of nodes. The results show that, as expected, most collisionavoidance protocols have lower efficiency than collisiondetection protocols. Nevertheless, there are exceptions, due to compatibility issues.

This paper is organized as follows. Section II describes the basic operation of the selected home network protocols. Section III presents mathematical analyses and Section IV reports simulation results for the selected protocols. Finally, concluding remarks and future directions are presented in Section V.

\section{Home Network Protocols}

The following subsections overview MAC sub-layers and physical layers of Ethernet, HomePNA, IEEE 802.11, and HomePlug. This information is used to calculate the maximum throughput and efficiency in Sections III and IV. The reader is referred to [2], [11], [12], [13], [10], [3], [4], [6], [14] and references therein for protocol details.

\section{A. Ethernet}

Ethernet uses Carrier Sense Multiple Access with Collision Detection (CSMA/CD) to control medium access. Before transmitting, the station senses the medium. If it is idle, after an inter-frame gap the station transmits the frame. If the medium is busy, the station keeps listening to the medium until it is idle and then, after an inter-frame gap, starts the frame transmission. During transmission, the station senses the medium to detect collisions. If a collision is detected, the station stops transmission and sends a jamming signal. Then, the station enters the binary exponential backoff phase. After the $n$th collision, the station waits for a random number of slot times, ranging from 0 to $2^{n}-1$, and then senses the medium.

Fig. 1 illustrates Ethernet frame format. The frame is composed of a preamble, destination and source addresses, a type field, data, and a Frame Check Sequence (FCS) using Cyclic Redundancy Check (CRC) [2]. If data length is less than 46 bytes, padding is used to fill 64 bytes, from destination address to FCS.

Ethernet has evolved in the last years. Higher speed specifications like Fast Ethernet and Gigabit Ethernet have come out [2]. These standards differ from basic Ethernet mainly in the physical layer but maintain frame format and minimum and maximum frame sizes keeping backward compatibility.

\section{B. HomePNA}

HomePNA 2.0 MAC is based on Ethernet CSMA/CD. HomePNA has an 8-level priority mechanism for QoS support. Different classes of traffic can be labeled with priorities from 0 to 7 , where 7 is the highest. Based on the frame priority, the transmission occurs in a specific time interval after an InterFrame Gap (IFG) of $29 \mu \mathrm{s}$, as shown in Fig. 2.

Time intervals are organized in decreasing order of priority. Higher priority frames are transmitted earlier not contending with lower priority ones. The duration of each priority slot, PRI_SLOT, is $21 \mu \mathrm{s}$. Stations must transmit their frames at the beginning of the slot whose number is equal to or lower than the frame priority. Any transmission after slot 0 is considered to happen at slot 0 .

Before transmission, the station senses the carrier and defers transmission if any carrier is detected before the time slot associated to the frame priority. In this case, time slot counting is restarted after the medium is idle and after an IFG.

All stations monitor the medium to detect collisions of frames transmitted by others. A collision can be detected through the transmission duration. The minimum duration of a valid frame is $92.5 \mu$ s whereas the maximum is $3122 \mu \mathrm{s}$. Any station that detects a collision ceases transmitting no later than $70 \mu$ s after the beginning of the frame.

If there is a collision, all stations start a distributed collision resolution algorithm called Distributed Fair Priority Queuing (DFPQ) [28]. After the algorithm execution, all stations involved in the collision are ordered in Backoff Levels (BL), which indicate the order these stations will transmit. The desired outcome is for only one station to be at BL 0 , enabling this station to access the channel. After a successful transmission, all other stations decrement their BLs, and new station(s) at BL 0 attempt transmission. All stations, even the ones not involved in the collision resolution procedure, monitor the medium activity to keep track of the Maximum Backoff Level (MBL). By monitoring the MBL, stations with frames that did not collide are not allowed to contend for access until all collided frames are transmitted successfully. The only exception is when a station has a frame with priority higher than the priority slot where the collision occurred. All stations must have eight BLs and eight MBL counters, one for each priority. 


\begin{tabular}{|c|c|c|c|c|c|}
\hline 8 & 6 & 6 & 2 & $>=46$ & 4 \\
\hline Preamble & $\begin{array}{l}\text { Destination } \\
\text { Address }\end{array}$ & $\begin{array}{c}\text { Source } \\
\text { Address }\end{array}$ & Type & Data & FCS \\
\hline
\end{tabular}

Fig. 1. Ethernet frame format.

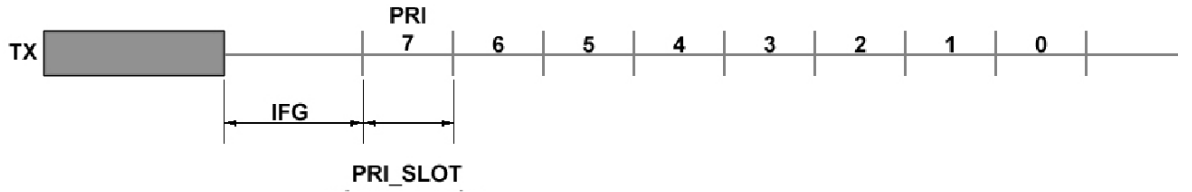

Fig. 2. HomePNA priority slots.

As shown in Fig. 3, after a collision occurs, there are 3 collision resolution signaling slots, numbered from S0 to S2, before the priority slots. BL and MBL counters are determined using the signaling slots, which have a duration of $32 \mu \mathrm{s}$.

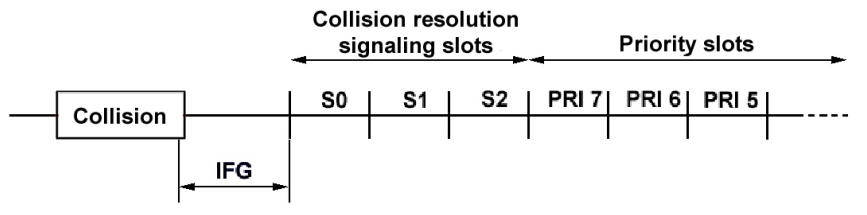

Fig. 3. Collision resolution signaling slots.

After a collision, the stations involved in collision resolution randomly choose a signaling slot to transmit a backoff signal. More than one station may transmit a signal in the same slot. If a station involved in the collision listens a backoff signal in a slot before the one the station has chosen, the station increments its BL counter. On the other hand, MBL counter is incremented for each backoff signal listened and decremented for each successful transmission. Therefore, MBL counter is non-zero whenever a collision resolution cycle is in progress. Stations not involved in the collision keep their BL counters equal to the MBL counters. These stations only transmit after the collision resolution completes.

HomePNA can adaptively use payload transmission rates from 4 to $32 \mathrm{Mbps}$, according to channel conditions. Nevertheless, the header and trailer are always transmitted at $4 \mathrm{Mbps}$, with more robust modulation and symbol rate to guarantee that all stations receive these fields correctly. The HomePNA frame is illustrated in Fig. 4.

The HomePNA frame is based on IEEE 802.3. Ethernet frame is preceded by a preamble and a frame control field, and followed by CRC, padding, and end-of-frame fields. Padding is used when transmission time of the complete frame is less than $92.5 \mu \mathrm{s}$, to guarantee minimum valid-frame duration.

1) HomePNA 3.0: HomePNA 3.0 supports synchronous (SMAC) and asynchronous (AMAC) medium access control. HomePNA 2.0 reaches $32 \mathrm{Mbps}$ data rates, whereas HomePNA 3.0 reaches $128 \mathrm{Mbps}$, with a $240 \mathrm{Mbps}$ extension [29].
Synchronous mode offers deterministic quality of service, which cannot be guaranteed in HomePNA 2.0. SMAC uses master-slave operation with admission control and resource reservation. Moreover, SMAC also aggregates packets to improve MAC efficiency [30], [31].

Asynchronous mode is compatible with HomePNA 2.0. Transmission rates can reach up to $128 \mathrm{Mbps}$ by using different QAM (Quadrature Amplitude Modulation) constellations and higher bandwidth. Nevertheless, basic transmission rate for header fields and EOF is 4 Mbps. Moreover, AMAC mode does not use packet aggregation, keeping the maximum frame size equal to 1500 bytes. The second difference to HomePNA 2.0 is a new collision management. Each node is assigned a set of three predefined collision resolution slots, called A, B, and C. The collision management guarantees that two nodes do not use the same set. Each slot A, B, or C can be defined as one of existing collision resolution slots $\mathrm{S} 0, \mathrm{~S} 1$, or S2. When a collision occurs, the node will use the first slot from its set (A). If a second collision happens for the same frame, the node will use slot B. In case of a third collision, slot $\mathrm{C}$ is used. As there is no slot sets repetition, each frame will collide at most three times, and after the third collision, every frame will be transmitted. This technique reduces the number of collisions and improves efficiency, but limits the number of nodes to 27 , the number of different sets.

Fig. 5 illustrates a collision resolution process between 27 nodes. Stations are labeled from $\mathrm{H} 0$ to $\mathrm{H} 26$, whereas $\mathrm{C} 1$ to $\mathrm{C} 13$ are collisions, numbered in order of occurrence. Slot sets for each node are represented in the collision sequence. For example, the set of station $\mathrm{H} 15$ is (S1, S2, S0). Note that a collision is resolved in three levels at most, guaranteeing that no frame collides more than three times. This is different from HomePNA 2.0 where there is a probability that frames collide indefinitely.

\section{IEEE 802.11}

IEEE 802.11 specifies two medium access algorithms: Distributed Coordination Function (DCF) and Point Coordination Function (PCF). DCF is a distributed mechanism, in which 


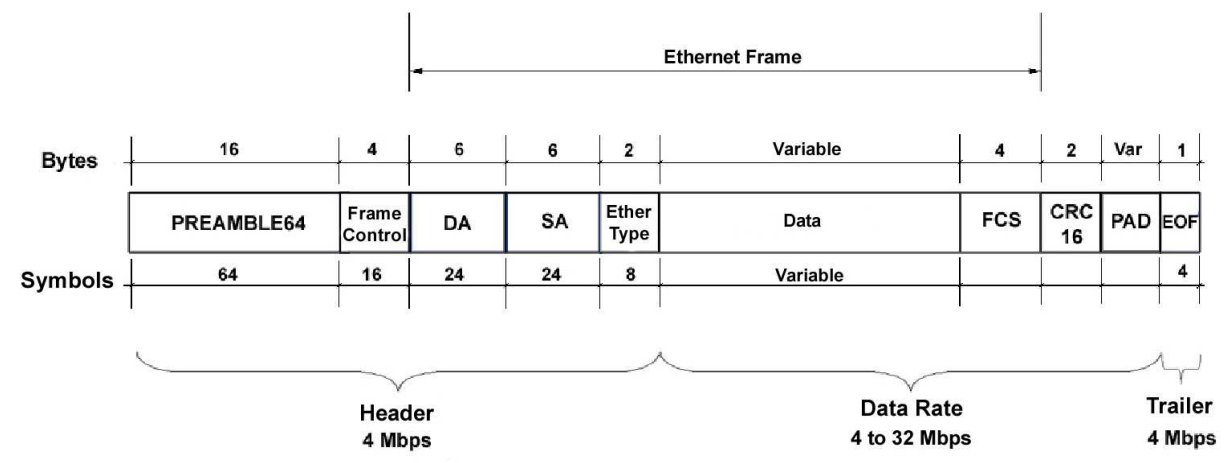

Fig. 4. HomePNA frame format.

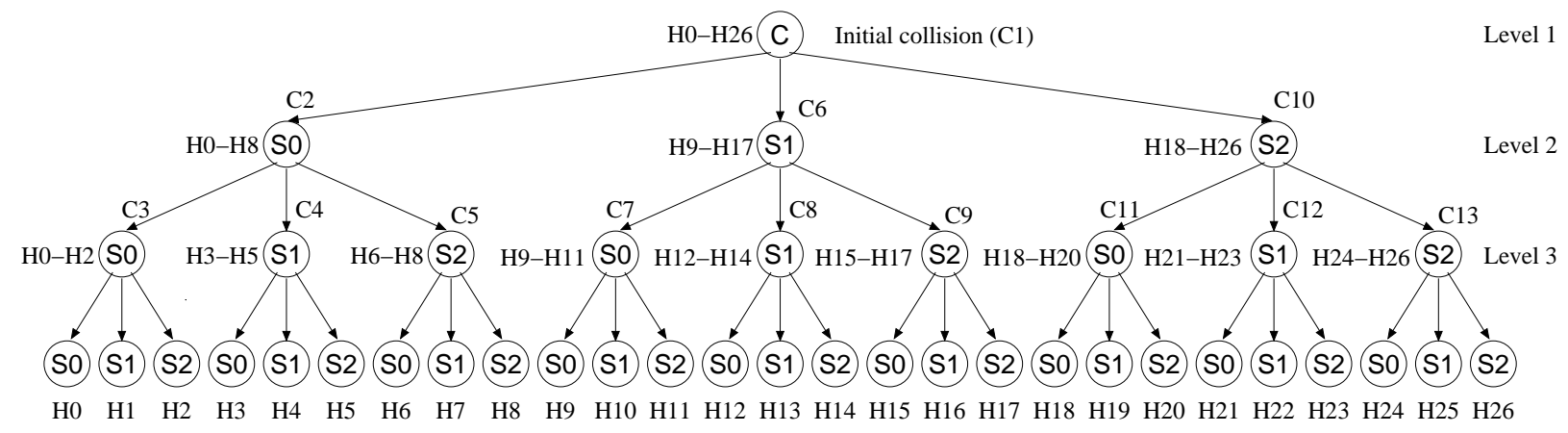

Fig. 5. Collision resolution between 27 nodes in HomePNA 3.0.

is idle. On the other hand, PCF is a centralized mechanism, where an access point controls medium access. Therefore, this mechanism is designed for infrastructure networks.

DCF operation uses Carrier Sense Multiple Access with Collision Avoidance (CSMA/CA) and positive acknowledgments (Fig. 6). Collision avoidance is used in wireless networks because it may not be possible to detect collisions in free space. Successful reception is not guaranteed because stations may not sense collisions at the receiver. In DCF, a station that wants to transmit first senses the medium. If it is idle for at least a period called Distributed Inter-Frame Space (DIFS), the station transmits. Else, transmission is postponed and a backoff is initiated. The station chooses a random number distributed between zero and the Contention Window (CW) size and starts a backoff timer. This timer is periodically decremented by a slot time each time the medium is idle for more than DIFS. Backoff timer is paused when a transmission is detected. If the medium is idle for another DIFS, the station resumes the backoff timer. When it expires, the station transmits.

The receiver uses CRC to detect errors. If the frame seems to be correct, the receiver sends an acknowledgment (ACK), after the medium is idle for a Short Inter-Frame Space (SIFS). By definition, SIFS is smaller than DIFS. If the sender does not receive an ACK, it schedules a retransmission and enters backoff. To reduce collision probability, the contention window starts with a minimum value $\mathrm{CW}_{\text {min }}$. After each unsuccessful attempt, the contention window increases to next power of 2 minus 1 , until reaching the maximum predefined value $\mathrm{CW}_{\max } . \mathrm{CW}_{\min }$ and $\mathrm{CW}_{\max }$ depend on the physical layer. Moreover, after a maximum number of retransmissions the frame is dropped. To avoid medium capture, before transmitting another frame the sending station will wait for DIFS and then enter the backoff phase. DCF method also optionally uses Request to Send (RTS) and Clear to Send (CTS) frames to avoid the hidden terminal problem [3].

IEEE 802.11 data frame is illustrated in Fig. 7. The frame is composed of frame control, duration, three addresses, sequence number, data, and FCS fields. Only three addresses are used in a fully connected ad hoc network. The data frame may include a fourth address in other configurations. ACK frames have frame control, duration, one address, and FCS fields.

Standard IEEE 802.11 operates in the $2.4 \mathrm{GHz}$ band and supports 1 and $2 \mathrm{Mbps}$ data rates. IEEE 802.11b [4] also uses $2.4 \mathrm{GHz}$ and supports up to $11 \mathrm{Mbps}$ using DSSS (Direct Sequence Spread Spectrum). IEEE 802.11a [5] uses the $5 \mathrm{GHz}$ band and defines up to $54 \mathrm{Mbps}$ data rates using OFDM (Orthogonal Frequency Division Multiplexing). IEEE 802.11g [6] uses OFDM in the $2.4 \mathrm{GHz}$ band and supports $54 \mathrm{Mbps}$.

Physical layer is composed of two sub-layers: a convergence sub-layer and a medium-dependent sub-layer. The convergence sub-layer is supported by the Physical Layer Convergence Protocol (PLCP). Different PLCPs are defined for each IEEE 802.11 extension.

IEEE 802.11 extensions have short and long PLCP Protocol Data Units (PPDUs). Long PPDUs are used for backward compatibility. The long PPDU for the $11 \mathrm{Mbps}$ HR-DSSS (High Rate - DSSS) $802.11 \mathrm{~b}$, which is mandatory, is shown 


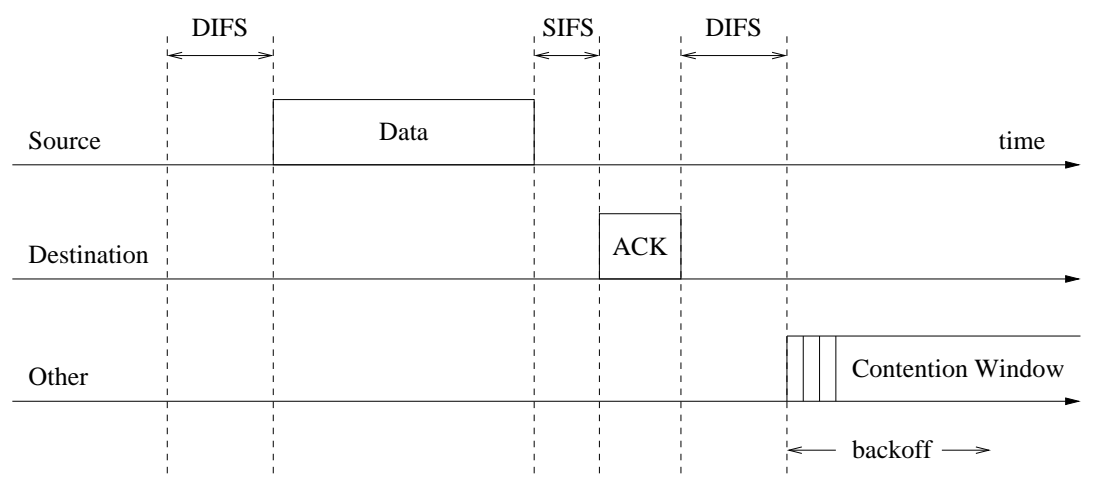

Fig. 6. Transmission of an IEEE 802.11 data frame.

\begin{tabular}{|c|c|c|c|c|c|c|c|}
\hline Bytes & 2 & 6 & 6 & 6 & 2 & $>=0$ & 4 \\
\hline $\begin{array}{l}\text { Frame } \\
\text { Control }\end{array}$ & Duration & Address & Address & Address & Sequence & Data & FCS \\
\hline
\end{tabular}

Fig. 7. IEEE 802.11 frame format.

\begin{tabular}{|c|c|c|} 
Bytes & \multicolumn{2}{c}{6} \\
\hline PLCP Preamble & PLCP Header & PSDU \\
\hline & $192 \mu \mathrm{s}$ \\
\hline \\
$1 \mathrm{Mbps}$
\end{tabular}

Fig. 8. Long PLCP PPDU for 11 Mbps HR-DSSS 802.11b.

in Fig. 8.

PPDU for $802.11 \mathrm{~g}$ using the $54 \mathrm{Mbps}$ ERP-OFDM (Extended Rate PHY - OFDM) is shown in Fig. 9.

\section{HomePlug}

Similarly to IEEE 802.11, HomePlug 1.0 uses CSMA/CA. Collision avoidance is used because it may not be possible to detect collisions in the electrical wiring, since the attenuation and noise can produce signal variations similar to collisions [27].

For QoS provision, the standard defines four priority levels. These levels are assigned according to the type of traffic, as standardized in IEEE 802.1D [32]. Priorities are associated to channel access classes ranging from $\mathrm{CA} 0$ to $\mathrm{CA} 3$, where $\mathrm{CA} 3$ is the highest.

Stations sense the medium before transmitting a data frame. To determine if the medium is busy, stations use Physical Carrier Sense (PCS) and Virtual Carrier Sense (VCS). Using only PCS, a node cannot be sure of whether there is another ongoing transmission or not [27]. The physical layer reports the physical carrier sense by detecting preambles or priority slot assertions. The MAC sub-layer uses virtual carrier sense to determine the transmission duration of the frame "listened" and to establish an allocation vector. Stations only contend for the medium after the expiration of their allocation vectors.

When the medium is idle for CIFS (Contention distributed Inter-Frame Space), a time interval of $35.84 \mu \mathrm{s}$, the station enters the priority resolution phase. Otherwise, if the station has been waiting for CIFS and the medium becomes busy, it waits for the medium to become idle for another CIFS. Two time slots are used during priority resolution (PR) assertions, in order to restrict the contention period only to stations with higher priority flows (Fig. 10).

Priority resolution is done before the contention period, using Priority Resolution Signals (PRS). PRSs use on-off modulation, where the number of each class is represented by a binary signal sent at the priority resolution periods, PR0 (Priority Resolution 0) and PR1 (Priority Resolution 1) [33]. Therefore, when a bit 1 is sent at PR0, every station with frames from classes lower than CA2 postpone their transmission, and wait for the medium to become idle for another CIFS. PR0 and PR1 time slots have the same duration of CIFS.

During contention, a station chooses a random number uniformly distributed between zero and the Contention Window (CW) size. This number is used as a backoff counter and will be decreased whenever the medium is idle. The backoff counter is decremented by one when the medium is idle for a time slot of $35.84 \mu \mathrm{s}$. Similarly to IEEE 802.11, the backoff procedure is responsible for increasing the contention window. The CW size depends on the number of times the backoff procedure has been called during the transmission of a frame. The backoff procedure is called every time a transmission fails or when, during backoff, a Deferral Counter (DC) reaches zero and the station senses another ongoing transmission. The Deferral Counter is a mechanism conceived to avoid collisions. It is decremented whenever a contending station determines that the medium has been captured by another station with the same priority. When DC reaches zero, the node assumes that there is a large number of stations trying to transmit and therefore the collision probability is high. In that case, the 


\begin{tabular}{|c|c|c|c|c|c|c|c|c|}
\hline 4 & 1 & 12 & 1 & 6 & 16 & \multicolumn{3}{|c|}{6} \\
\hline Rate & Reserved & Length & Parity & Tail & Service & PSDU & Tail & Pad Bits \\
\hline \multirow[t]{2}{*}{ Symbols } & 12 & 6 & \multicolumn{6}{|c|}{ Variable } \\
\hline & PLCP Preamb & Signal & \multicolumn{6}{|c|}{ Data } \\
\hline
\end{tabular}

Fig. 9. PLCP PPDU for 54 Mbps ERP-OFDM 802.11g.

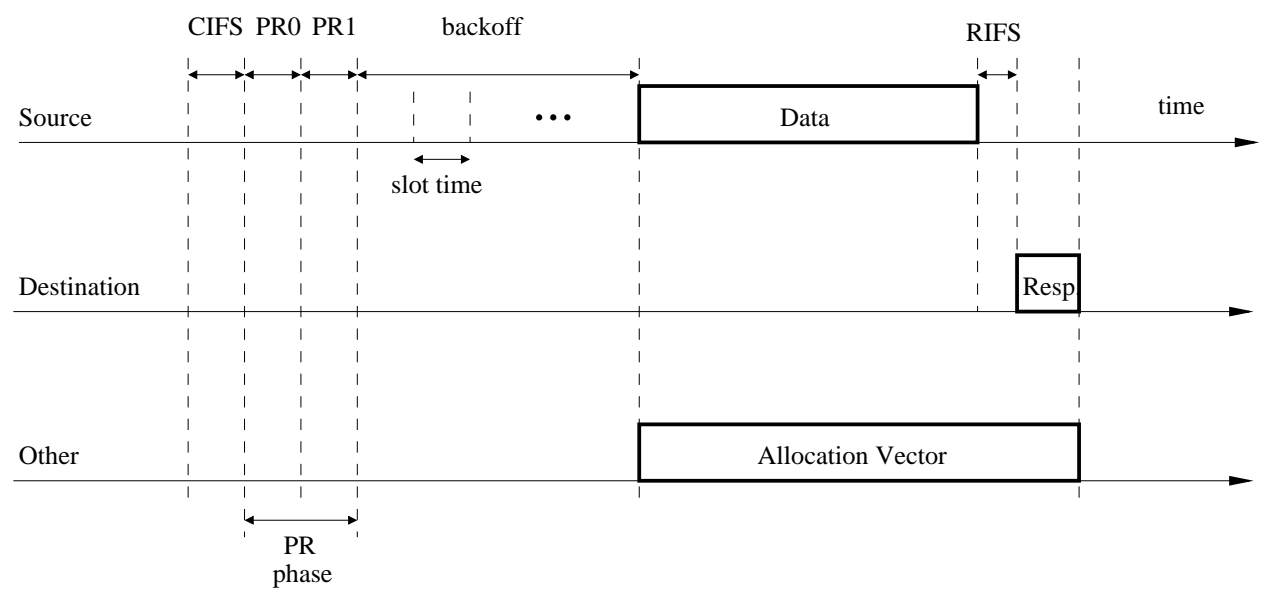

Fig. 10. Transmission of a HomePlug data frame.

station calls the backoff procedure.

Upon reception of a frame, the receiver checks if the transmitter waits a response. If it does, the receiver waits for RIFS (Response Inter-Frame Space) before sending a response. The response can be an ACK, when a well-succeeded reception occurs; a NACK (Negative Acknowledgment), when an error has been detected but could not be corrected; or FAIL, if a frame could not be stored due to lack of buffer space.

The electrical wiring may irradiate as an antenna. Hence, privacy is an important issue and must be taken into account by HomePlug. HomePlug uses an 8-byte block size encryption algorithm, which is applied over the ether type, data and ICV fields (Fig. 11). The ECtl field defines the encryption parameters and the EPad field is needed to guarantee that the encrypted portion is a multiple of 8 bytes.

The HomePlug data frame is presented in Fig. 11.

HomePlug 1.0 uses a spectral band that goes approximately from 4.49 to $20.7 \mathrm{MHz}$. HomePlug uses OFDM dividing the band from 0 to $25 \mathrm{MHz}$ into 128 subcarriers evenly spaced, from which only 84 are used. Additionally, other 8 subcarriers may be disabled to avoid interference with amateur bands, leaving only 76 subcarriers for utilization. The duration of the OFDM symbol is $8.4 \mu \mathrm{s}$.

The payload consists of a number of blocks with 20 or 40 OFDM symbols each, encoded on a link-by-link basis using a Reed-Solomon code concatenated with a convolutional code. The division that generates these block sizes is used to avoid impulsive noise that can damage symbol sequences. The convolutional encoder has constraint length 7 and code rates of $\frac{1}{2}$ or $\frac{3}{4}$, selected during the channel adaptation. The ReedSolomon code, which is used after the convolutional code, has coding rates ranging from $\frac{23}{39}$ to $\frac{238}{254}$.

Assuming the parameters described above, the physical layer can offer up to 139 different rate combinations, ranging from 1 to 14 Mbps.

Additionally, there is a mode called ROBO (ROBust OFDM). This mode has greater redundancy to operate under noisy situations. It uses DBPSK (Differential Binary Phase Shift Keying) modulation, with a redundancy level that reduces the rate to $\frac{1}{4} \mathrm{bit} / \mathrm{symbol} / \mathrm{subcarrier}$. It also uses a ReedSolomon code with different code rates that range from $\frac{31}{39}$ to $\frac{43}{51}$. These parameters reduce the maximum transmission rate to $0.9 \mathrm{Mbps}$.

\section{Mathematical ANALysis}

Home network applications, like video, demand high transmission rates. However, physical layer rate is not the most appropriate parameter for analyzing network suitability for these applications. MAC protocol throughput must be taken into account. This section provides the mathematical analysis of the one-node maximum throughput that can be obtained using four different home network technologies. We make the following assumptions: there is a single sender and a single receiver; bit error rate is zero; propagation delay is negligible; the source always has a frame ready for transmission; no fragmentation. The analysis uses the notations presented in Table I.

The throughput $(T h)$ is calculated by dividing the size of the MAC SDU (Service Data Unit) by its transmission time $(T)$. Depending on MAC SDU size, padding may be used. 


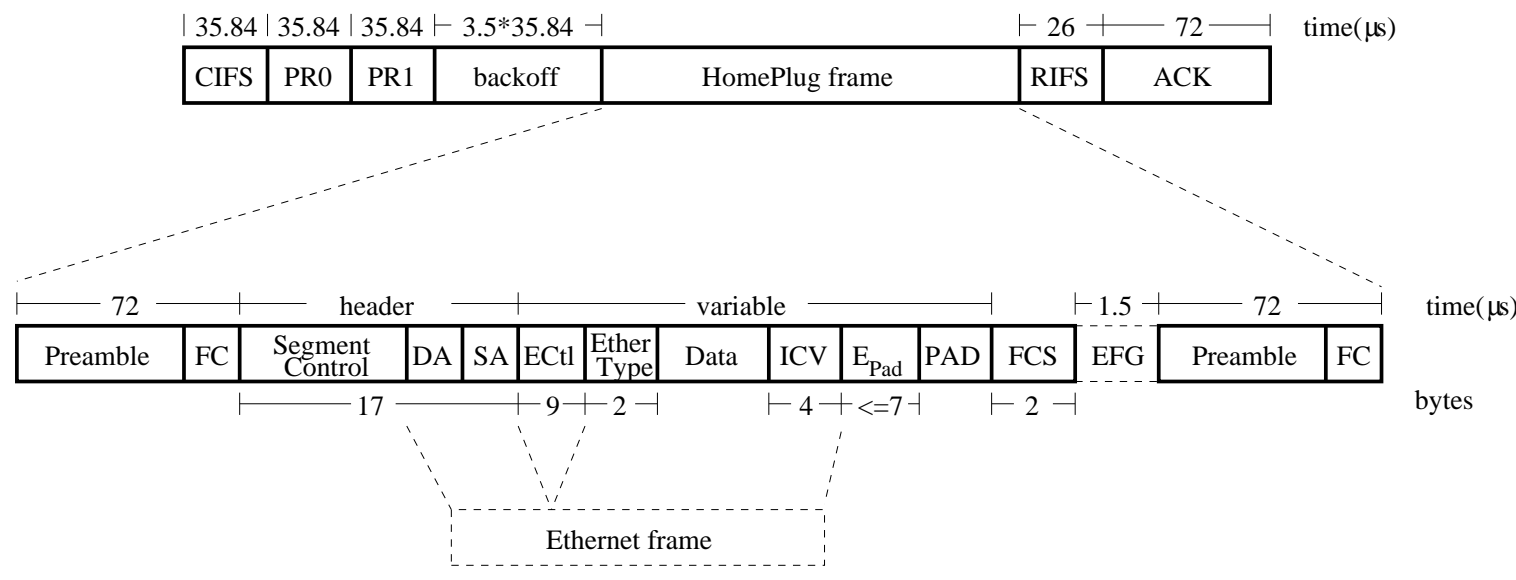

Fig. 11. HomePlug frame format.

TABLE I

NOTATIONS USED FOR MATHEMATICAL ANALYSIS.

$C_{E R R} \quad$ Error correction code rates.

$C W_{\min }$ Minimum contention window size.

$L_{A C K} \quad$ ACK size (in bytes).

$L_{D A T A}$ Payload size (in bytes).

$L_{E P a d} \quad$ Encryption padding size (in bytes).

$L_{I F G} \quad$ Inter-frame gap size (in bits).

$L_{P A D} \quad$ Padding size (in bytes).

$N_{B S S} \quad$ Number of bits per symbol per subcarrier (in bits).

$N_{D B S} \quad$ Number of data bits per symbol (in bits).

$N_{S C} \quad$ Number of subcarriers.

$N_{S Y M} \quad$ Number of symbols.

$N_{S P B} \quad$ Number of symbols per block.

$P \quad$ Priority.

$R_{C T L} \quad$ Physical control rate (in Mbps).

$R_{D A T A} \quad$ Physical data rate (in Mbps).

$T_{A C K} \quad$ Transmission time of the acknowledgment (in $\mu \mathrm{s}$ ).

$T_{C I F S} \quad$ CIFS time (in $\mu \mathrm{s}$ ).

$T_{D I F S} \quad$ DIFS time (in $\mu \mathrm{s}$ ).

$T_{E F G} \quad$ Transmission time of the end of frame gap (in $\mu \mathrm{s}$ ).

$T_{E X T} \quad$ Signal extension (in $\mu \mathrm{s}$ ).

$T_{I F G} \quad$ Transmission time of the inter-frame gap (in $\mu \mathrm{s}$ ).

$T_{P H Y} \quad$ Transmission time of the physical preamble and header (in $\mu$ s).

$T_{P R} \quad$ Priority resolution time (in $\mu \mathrm{s}$ ).

$T_{R I F S} \quad$ RIFS time (in $\mu \mathrm{s}$ ).

$T_{\text {slot }} \quad$ Slot time (in $\mu \mathrm{s}$ ).

$T_{S I F S} \quad$ SIFS time (in $\mu \mathrm{s}$ ).

$T_{S Y M} \quad$ Transmission time of a symbol (in $\mu \mathrm{s}$ ).

\section{A. Fast Ethernet}

First, we analyze the maximum throughput of Ethernet. According to Fig. 1, the total transmission time of an Ethernet frame is

$$
T_{\text {Ether }}=\frac{\left(L_{D A T A}+L_{P A D}+26\right) \times 8+L_{I F G}}{R_{D A T A}} \mu s .
$$

If $L_{D A T A}<46, L_{P A D}=46-L_{D A T A}$, else $L_{P A D}=0$. For Fast Ethernet, $R_{D A T A}=100 \mathrm{Mbps}, L_{I F G}=96$ bits, and the throughput is given by

$$
T h_{\text {Ether } 100}=\frac{L_{D A T A} \times 8}{\frac{304+8 \times\left(L_{D A T A}+L_{P A D}\right)}{100}} \text { Mbps. }
$$

The throughput can be as low as 72.46 Mbps using a payload size of 100 bytes and as large as 97.53 Mbps for 1500-byte frames.

\section{B. HomePNA 2.0 and 3.0}

In the computation of HomePNA maximum throughput we only consider HomePNA 2.0 and HomePNA 3.0 AMAC, because SMAC uses a Master-Slave configuration where there is no contention.

Based on Figs. 2 and 4, the total transmission time of a HomePNA frame is

$$
\begin{aligned}
T_{H P N A}= & T_{I F G}+(7-P) \times 21+\frac{35 \times 8}{4}+ \\
& \frac{\left(L_{D A T A}+L_{P A D}+6\right) \times 8}{R_{D A T A}} \mu s . \\
T_{H P N A}= & 29+(7-P) \times 21+70+ \\
& \frac{\left(L_{D A T A}+L_{P A D}+6\right) \times 8}{R_{D A T A}} \mu s .
\end{aligned}
$$

Then the throughput for HomePNA is

$T h_{H P N A}=\frac{L_{D A T A} \times 8}{246-21 \times P+\frac{48+8 \times\left(L_{D A T A}+L_{P A D}\right)}{R_{D A T A}}}$ Mbps.

If the frame transmission time is lower than $92.5 \mu \mathrm{s}, L_{P A D}$ is the smallest number that guarantees that the transmission time is at least $92.5 \mu \mathrm{s}$.

HomePNA 2.0 achieves a throughput of 6.37 Mbps using frames with 100 bytes of payload, and 25.24 Mbps using 1500byte frames, considering the highest priority. 
For HomePNA 3.0 AMAC using priority 7, the throughput for 100-byte frames reaches $7.57 \mathrm{Mbps}$ whereas with 1500byte frames the throughput is $62.14 \mathrm{Mbps}$, for a $128 \mathrm{Mbps}$ PHY rate.

\section{IEEE 802.11}

In the analysis of IEEE 802.11, we consider the basic access mechanism (DCF) using 802.11b and 802.11g. The analysis can be easily extended to RTS/CTS mechanism and to other extensions.

For IEEE $802.11 \mathrm{~b}$, according to Figs. 6, 7, and 8, the transmission time of a frame is

$$
\begin{aligned}
T_{802.11 b}= & T_{D I F S}+\frac{C W_{\text {min }}}{2} \times T_{\text {slot }}+T_{P H Y}+ \\
& \frac{\left(L_{D A T A}+28\right) \times 8}{R_{D A T A}}+T_{S I F S}+T_{P H Y}+ \\
& \frac{L_{A C K} \times 8}{R_{C T L}} \mu s .
\end{aligned}
$$

Replacing the values for IEEE $802.11 \mathrm{~b}$ using $11 \mathrm{Mbps}$ HRDSSS [4], Equation 6 becomes

$$
\begin{aligned}
T_{802.11 b}= & 50+\left(\frac{31}{2} \times 20\right)+192+\frac{\left(L_{D A T A}+28\right) \times 8}{11} \\
& +10+192+\frac{14 \times 8}{1} \mu s .
\end{aligned}
$$

Then, the throughput for IEEE $802.11 \mathrm{~b}$ is

$$
T h_{802.11 b}=\frac{L_{D A T A} \times 8}{866+\frac{224+8 \times L_{D A T A}}{11}} \text { Mbps. }
$$

Using Equation 8, the throughput of $11 \mathrm{Mbps} 802.11 \mathrm{~b}$ is $0.83 \mathrm{Mbps}$ for 100-byte frames and $6.07 \mathrm{Mbps}$ for 1500-byte frames.

For ERP-OFDM 802.11g, according to Figs. 6, 7, and 9, and using a ceiling function to account for padding bits, the total frame transmission time is

$$
\begin{aligned}
T_{802.11 g}= & T_{D I F S}+\frac{C W_{\text {min }}}{2} \times T_{\text {slot }}+T_{P H Y}+ \\
& N_{S Y M} \times T_{S Y M}+T_{E X T}+T_{S I F S}+T_{P H Y} \\
& +\left\lceil\frac{16+8 \times L_{A C K}+6}{N_{D B S}}\right\rceil \times T_{S Y M}+ \\
& T_{E X T} \mu s .
\end{aligned}
$$

The number of symbols, $N_{S Y M}$, depends on the number of data bits per symbol, $N_{D B S}$, as shown in Equation 10.

$$
N_{S Y M_{802.11 g}}=\left\lceil\frac{16+8 \times\left(L_{D A T A}+28\right)+6}{N_{D B S}}\right\rceil .
$$

Replacing the values for $54 \mathrm{Mbps} 802.11 \mathrm{~g}$ [6], Equation 9 can be rewritten as

$$
\begin{aligned}
T_{802.11 g}= & 50+\frac{15}{2} \times 20+20+ \\
& \left\lceil\frac{16+8 \times\left(L_{D A T A}+28\right)+6}{216}\right\rceil \times 4+6+ \\
& 10+20+\left\lceil\frac{16+8 \times 14+6}{24}\right\rceil \times 4+ \\
& 6 \mu \mathrm{s} .
\end{aligned}
$$

Then, the throughput for IEEE $802.11 \mathrm{~g}$ is given by

$$
T h_{802.11 g}=\frac{L_{D A T A} \times 8}{286+\left\lceil\frac{246+8 \times L_{D A T A}}{216}\right\rceil \times 4} \text { Mbps. }
$$

Therefore, the throughput of IEEE $802.11 \mathrm{~g}$ varies from 2.61 Mbps using a payload size of 100 bytes to $23.35 \mathrm{Mbps}$ using 1500-byte payload, for a $54 \mathrm{Mbps}$ PHY rate.

\section{HomePlug 1.0}

Finally, in this section the throughput of HomePlug 1.0 is analyzed. According to Figs. 10 and 11, the time needed to transmit a HomePlug frame is

$$
\begin{aligned}
T_{H p l u g}= & T_{C I F S}+T_{P R}+\frac{C W_{\text {min }}}{2} \times T_{\text {slot }}+T_{P H Y}+ \\
& N_{S Y M} \times T_{S Y M}+T_{E F G}+T_{P H Y}+ \\
& T_{R I F S}+T_{A C K} \mu s .
\end{aligned}
$$

All stations must receive delimiters as well as priority resolution signals correctly, therefore they are sent using all subcarriers, with the same modulation and codification.

The number of symbols, $N_{S Y M}$, depends on the number of bits per symbol per subcarrier $N_{B S S}$, on the number of subcarriers $N_{S C}$, on the error correction codes $C_{E R R}$, and on the number of symbols per block $N_{S P B}$, as shown in Equation 14. Data are transmitted into 20 or 40 OFDM symbol transmission blocks. Thus, the number of blocks must be rounded up.

The number of symbols is given by

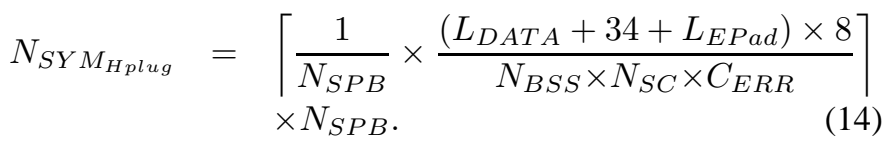

The encryption padding size is calculated as shown in Equation 15.

$$
L_{E P a d}=\left\lceil\frac{L_{D A T A}}{8 \times 8}\right\rceil \times 8-\frac{L_{D A T A}}{8} \text { bytes. }
$$

For maximum throughput, we have $N_{B S S}=2$ bits/symbol/subcarrier, $N_{S C}=84$ subcarriers, $C_{E R R}=$ $\frac{3}{4} \times \frac{238}{254}$, and $N_{S P B}=20$ symbols per block [34]. Then, Equation 13 is rewritten as

$$
\begin{aligned}
T_{\text {Hplug }}= & 35.84+2 \times 35.84+\frac{7}{2} \times 35.84+72+ \\
& \left\lceil\frac{1}{20} \times \frac{\left(L_{D A T A}+34+L_{E P a d}\right) \times 8}{118.06299}\right\rceil \times 20 \times \\
& 8.4+1.5+72+26+72 \mu \mathrm{s} .
\end{aligned}
$$


The throughput for HomePlug is given by

$$
T h_{H p l u g}=\frac{L_{D A T A} \times 8}{476.46+\left\lceil\frac{272+8 \times\left(L_{D A T A}+L_{E P a d}\right)}{2361.2598}\right\rceil \times 168} \text { Mbps. }
$$

Using this equation, HomePlug throughput is $1.24 \mathrm{Mbps}$ for 100-byte frames and $8.08 \mathrm{Mbps}$ for 1500-byte frames, for a 14 Mbps PHY rate.

Our mathematical analyses consider one sender and one receiver. In order to evaluate the throughput on more realistic scenarios with higher number of nodes, we use simulation, as described in the next section.

\section{Simulation Results}

Network simulator (ns-2) [35] has been used in the simulations. We have implemented modules for HomePNA and HomePlug in ns-2.

Simulations of different protocols are divided into two sets. The first simulations compare the throughput expected from mathematical analysis to the results obtained with simulation. The second simulation set analyzes the throughput for varying network sizes.

The offered load is produced by one node, which sends frames continuously, i.e., the node always has a frame to send as soon as the medium gets idle. In the payload graphs, theoretical results are represented by continuous lines whereas simulation results use points. Data payload ranges from 160 to 1500 bytes. Each simulation run lasts for 100 seconds.

For the second simulation set, the number of senders ranges from 1 to 30. Payload size is 1500 bytes. Again, each simulation run lasts for 100 seconds. To obtain maximum occupation, all senders try to transmit continuously. Thus, whenever the medium is idle, all stations try to transmit, collide, and start collision resolution. These simulations investigate the behavior of different MAC protocols when collisions happen. Graphs have vertical bars corresponding to a confidence interval of $98 \%$.

We evaluate the maximum throughput for Fast Ethernet, HomePNA 2.0 and 3.0, IEEE $802.11 \mathrm{~b}$ and $802.11 \mathrm{~g}$, and HomePlug 1.0. We also compute the efficiency of each protocol dividing its throughput by its respective physical data rate.

\section{A. Fast Ethernet}

Ethernet provided by ns-2 had to be modified in order to take the Ethernet preamble and CRC into account. Fig. 12 presents the maximum throughput of Fast Ethernet. As expected, the throughput increases with the payload size. Ethernet efficiency is as large as $97.5 \%$ for 1500 -byte payload. Moreover, the simulation model reproduces the behavior of the analytical model.

Then, performance of Fast Ethernet for varying number of nodes is measured. In Fig. 13, throughput decreases as the number of nodes increases, but even with 30 nodes transmitting simultaneously, throughput is higher than $70 \mathrm{Mbps}$, or $70 \%$ of the PHY data rate.

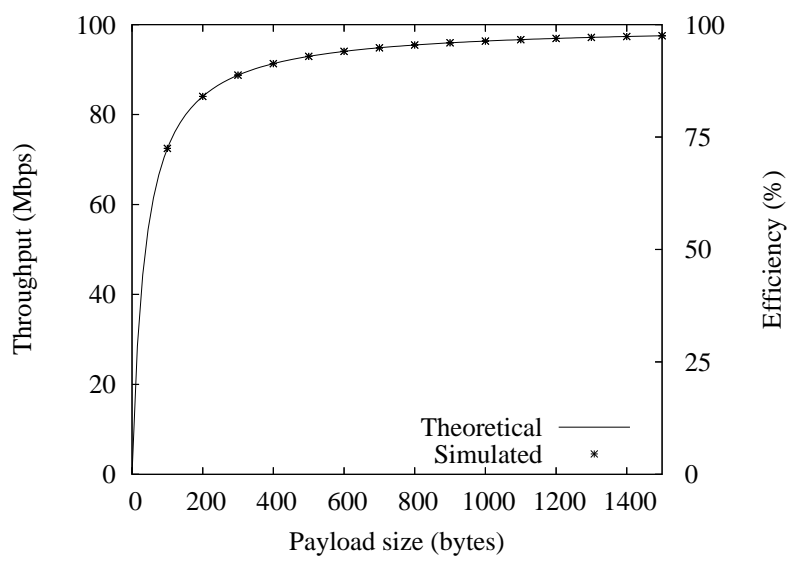

Fig. 12. Throughput of Fast Ethernet for different payload sizes.

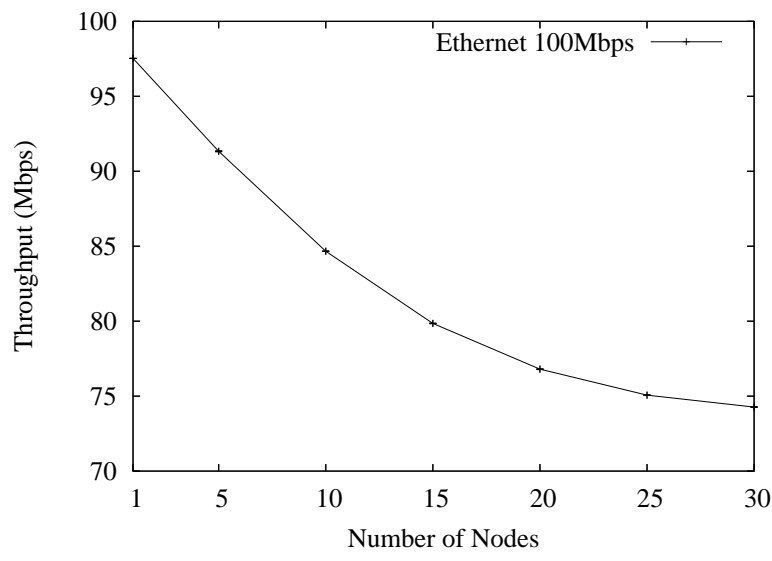

Fig. 13. Throughput of Fast Ethernet for varying number of sources.

\section{B. HomePNA}

We have implemented HomePNA 2.0 and 3.0 modules based on Ethernet available in ns-2. In addition to different access methods of HomePNA and Ethernet, the priority and collision resolution functionalities of HomePNA have been implemented [36]. Moreover, we have implemented a physical layer with $4 \mu$ s propagation delay, the same value used by Ethernet.

The first simulations have verified HomePNA operation. All stations transmit using highest priority, 7. Fig. 14 presents the throughput obtained for varying frame sizes. Note that the simulation results reproduce the mathematical model.

The second simulation set evaluates network throughput with 1 to 30 nodes and 1500-byte frames. Physical transmission rate is $32 \mathrm{Mbps}$. Fig. 15 plots the throughput obtained by HomePNA 2.0. Note that it tends to constant for a high number of nodes. This is due to the collision resolution algorithm of HomePNA, which produces a number of collisions proportional to the number of initially collided frames. For large number of nodes, a group of $3 n$ nodes that collided tend to be divided into three sets with $n$ nodes each. If each group of $n$ nodes collide $C$ times in average, the whole group (with $3 n$ nodes) collide $3 C+1$ times, which is $3 C$ for large $C$. Therefore, for large $n$, collision resolution is linear, i.e., the 


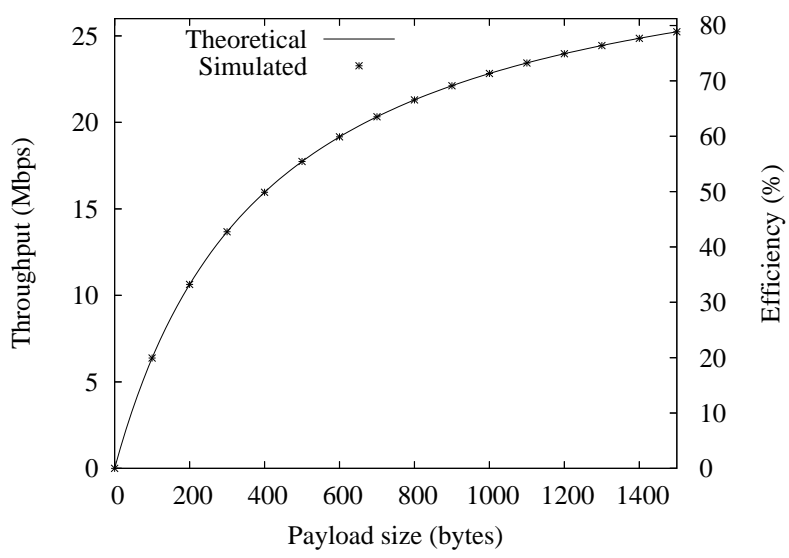

Fig. 14. Throughput of HomePNA 2.0 for different payload sizes.

number of collisions needed to solve the initial collision is proportional to the number of stations involved in it. Then, the throughput tends to constant for large number of nodes [36]. For 1500-byte frames and large number of nodes, aggregated throughput is $17.7 \mathrm{Mbps}$, or $55.3 \%$ of physical data rate.

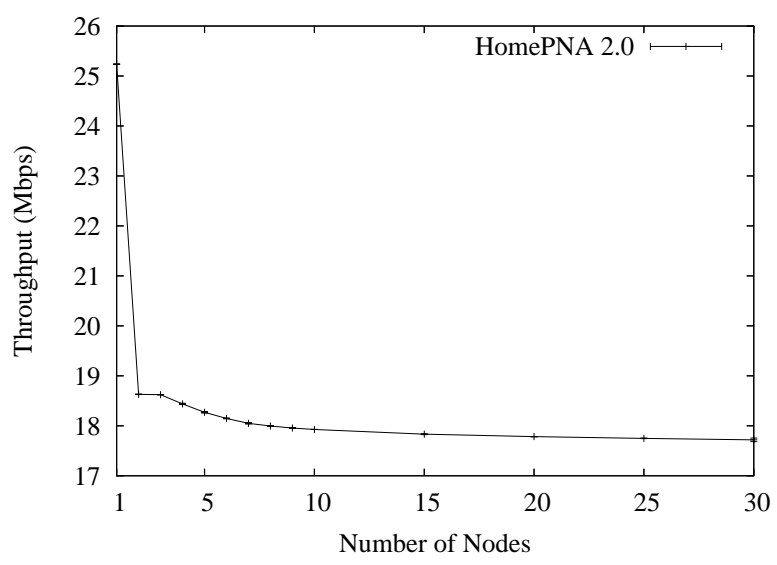

Fig. 15. Throughput of HomePNA 2.0 for varying number of sources.

1) HomePNA 3.0: For HomePNA 3.0, the number of nodes in the network varies from 1 to 27 , the maximum number of nodes allowed. Physical rate is $128 \mathrm{Mbps}$. Slot sets used for collision resolution are randomly chosen. All nodes have priority 7 to obtain maximum throughput.

Fig. 16 presents the throughput obtained by HomePNA 3.0 for varying frame size. Small frames yield small throughput, for 160-byte frames, as low as 5 Mbps. Maximum throughput is 62.1 Mbps using 1500-byte frames, for an efficiency of $48.5 \%$. The small efficiency is explained by backwardcompatible low basic rate used to transmit headers and end of frames.

Fig. 17 plots the throughput using 1500-byte frames and variable number of nodes. As opposed to HomePNA 2.0, where the throughput tends to a constant for large number of nodes, HomePNA 3.0 throughput increases with the number of nodes. This is due to the collision management protocol, which reduces collisions per frame for large numbers of nodes, as shown in [20].

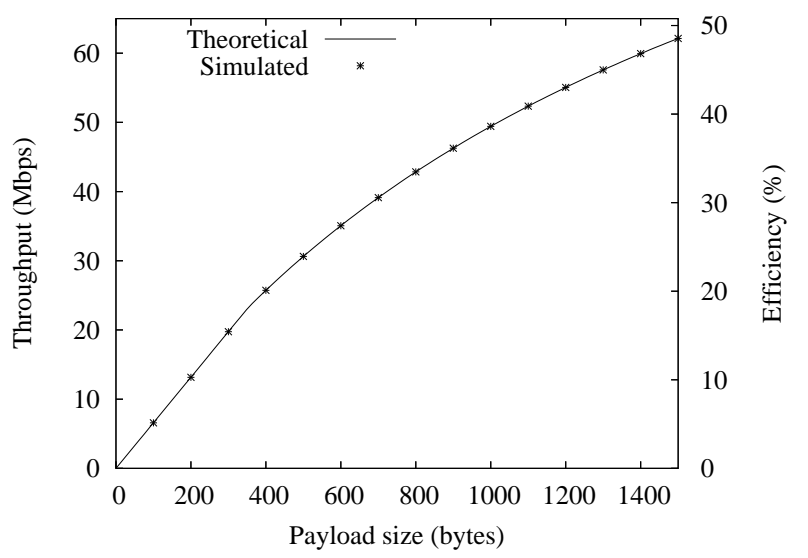

Fig. 16. Throughput of HomePNA 3.0 for different payload sizes.

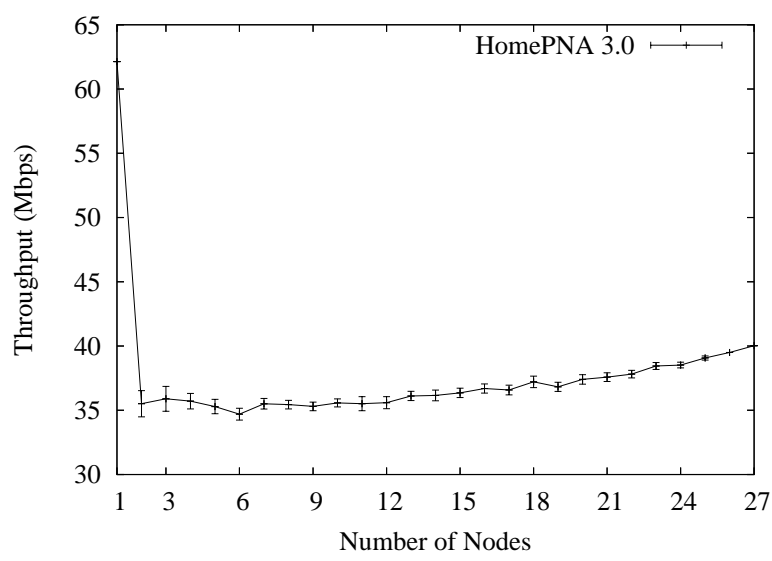

Fig. 17. Throughput of HomePNA 3.0 for varying number of sources.

Fig. 17 also shows a huge difference between physical rate and maximum throughput. For more than one node, the throughput falls to approximately half the one-node throughput, or 34.7 Mbps. In HomePNA 2.0, as soon as a collision is detected, the frame transmission stops. The same is valid for HomePNA 3.0. Nevertheless, to keep compatibility, HomePNA 3.0 uses the same collision resolution slot times and minimum frame duration as HomePNA 2.0. Thus, the time spent with one collision is longer than the time spent with the transmission of one frame at $128 \mathrm{Mbps}$ in HomePNA 3.0, reducing its efficiency.

\section{IEEE 802.11}

We have modified ns-2 to implement IEEE 802.11g. In the simulations, all stations are within transmission range. We used the free space propagation model to calculate attenuation.

First, we run simulations to evaluate the maximum throughput obtained by IEEE 802.11 for different payload sizes. All nodes are either $802.11 \mathrm{~b}$ nodes or $802.11 \mathrm{~g}$ nodes. We use IEEE $802.11 \mathrm{~b} 11 \mathrm{Mbps}$ HR-DSSS and IEEE $802.11 \mathrm{~g}$ 54 Mbps ERP-OFDM. Figs. 18 and 19 plot the maximum throughput for varying payload sizes using IEEE $802.11 \mathrm{~b}$ and $802.11 \mathrm{~g}$, respectively. Both $802.11 \mathrm{~b}$ and $802.11 \mathrm{~g}$ simulation results confirm the theoretical analysis. 
The maximum efficiency of IEEE $802.11 \mathrm{~b}$ is higher than IEEE $802.11 \mathrm{~g}$ one because its overhead is smaller. Using 1500 -byte frames, $802.11 \mathrm{~b}$ has maximum efficiency around $55 \%$, whereas $802.11 \mathrm{~g}$ efficiency is below $45 \%$. IEEE $802.11 \mathrm{~g}$ transmits data at $54 \mathrm{Mbps}$ with a basic rate of $6 \mathrm{Mbps}$ whereas $802.11 \mathrm{~b}$ uses $11 \mathrm{Mbps}$ and $1 \mathrm{Mbps}$, respectively. On the other hand, 802.11g uses the same SIFS time, slot time, and maximum CW. Only minimum $\mathrm{CW}$ value is reduced, from 31 to 15 . Nevertheless, the standard defines an optional extension called $802.11 \mathrm{~g}$ Short Slot Time, which provides higher throughput by reducing the slot time from 20 to $9 \mu \mathrm{s}$. With this extension the maximum efficiency of $802.11 \mathrm{~g}$ is $53 \%$.

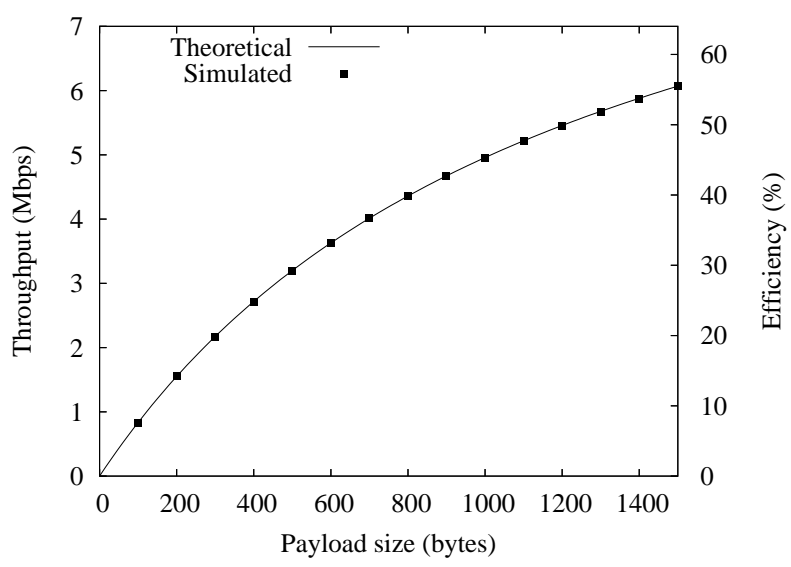

Fig. 18. Throughput of IEEE $802.11 \mathrm{~b}$ for different payload sizes.

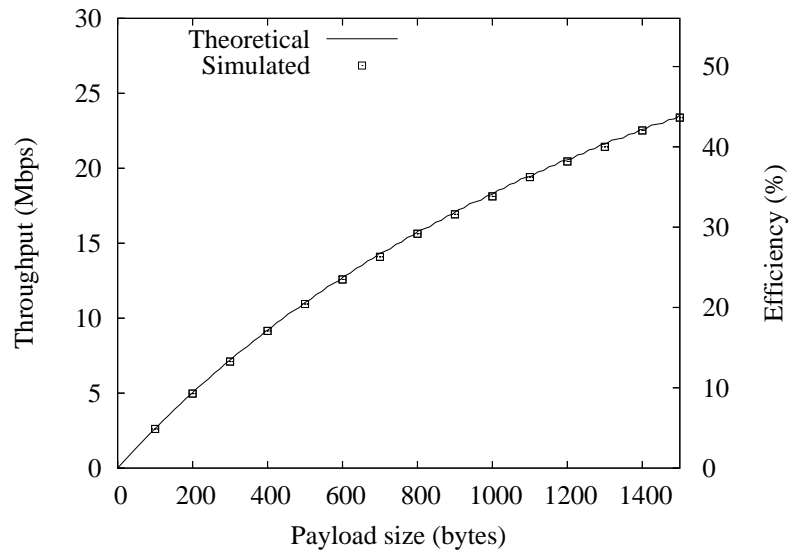

Fig. 19. Throughput of IEEE 802.11g for different payload sizes.

In the next simulations, the number of nodes is varied. Figs. 20 and 21 show the throughput obtained by IEEE $802.11 \mathrm{~b}$ and $802.11 \mathrm{~g}$, respectively. Frame size is 1500 bytes. As the number of nodes increases, throughput decreases due to more collisions. Note that the throughput increases from 1 to 3 sources, because the initial contention window $\left(C W_{\min }\right)$ size is too large, adding more idle slots than needed. Up to 3 sources, contention for the medium reduces the average number of idle slots increasing the throughput. For more than 3 sources, throughput decreases due to increasing collisions.

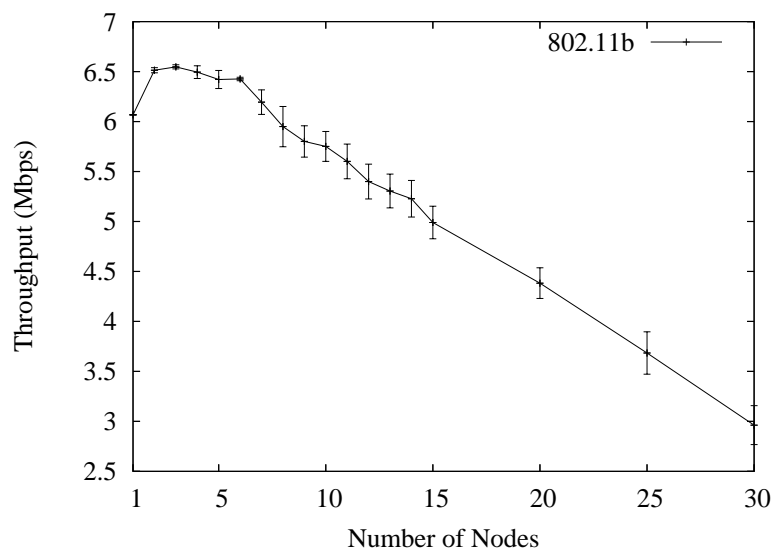

Fig. 20. Throughput of IEEE $802.11 \mathrm{~b}$ for varying number of sources.

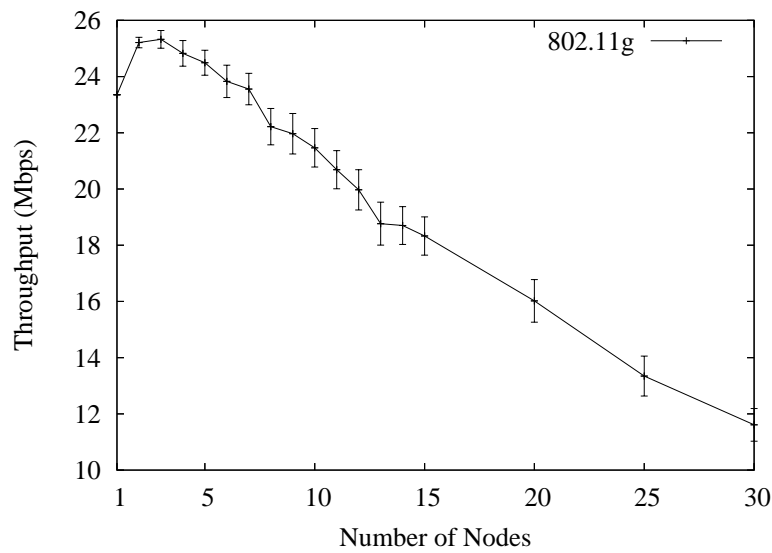

Fig. 21. Throughput of IEEE $802.11 \mathrm{~g}$ for varying number of sources.

\section{HomePlug 1.0}

We have implemented two HomePlug modules, a physical layer and a MAC sub-layer [37]. MAC module is based on HomePlug version 1.0. Our physical layer is based on the echo model introduced in [34]. Due to ramifications that an electrical network may have as well as reflections caused by impedance mismatches, the transmitted signal may be received through multiple paths. The echo sums up all the signals received, which may be out of phase and have different amplitudes. Simulations use the channel that presents the best behavior among the examples provided by Langfeld [38]. Our simulations use the maximum throughput parameters of Section III.

The source and receiver nodes are separated by 5 meters. Data transmission is $14 \mathrm{Mbps}$ and payload size varies. Fig. 22 shows that the throughput obtained confirms the mathematical analysis of Section III-D. The theoretical maximum throughput is saw-tooth shaped. The cause is the padding inserted to keep the number of symbols per frame a multiple of 20 . Periodic throughput falls happen when an additional symbol block is used. As the payload increases, padding decreases and throughput grows, until another block is needed.

Fig. 23 shows the maximum throughput varying the number of transmitters. Every node is transmitting at $14 \mathrm{Mbps}$ 


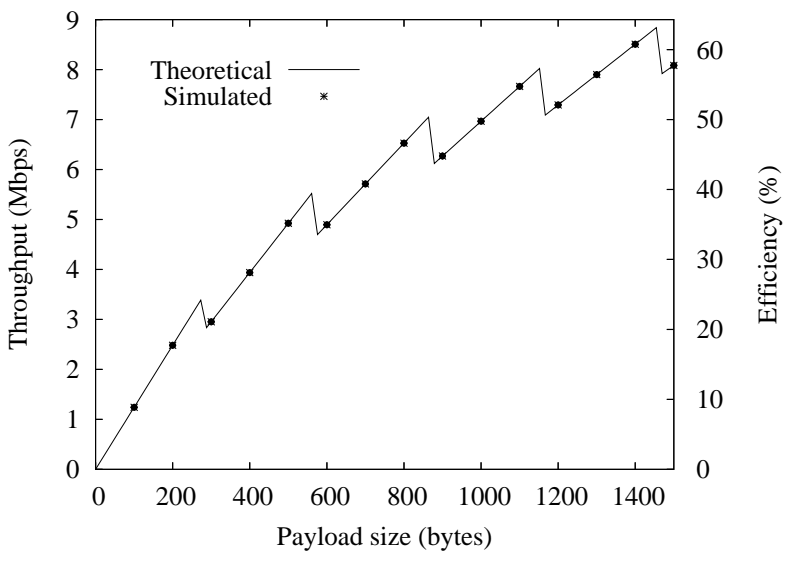

Fig. 22. Throughput of HomePlug for different payload sizes.

with equal priority. HomePlug limits the number of nodes to 16. More nodes are allowed only in ROBO mode. The throughput decrease is due to higher number of collisions. Collisions increase because the probability of more than two nodes choosing the same slot time increases with the number of nodes. Unlike IEEE 802.11, HomePlug throughput does not increase for a few nodes because its $C W_{\min }$ is small producing few idle slots.

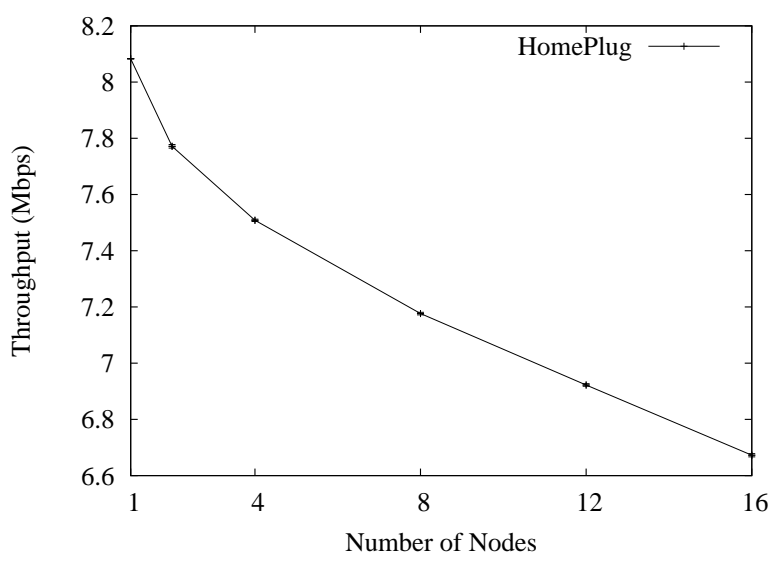

Fig. 23. Throughput of HomePlug for varying number of sources.

\section{E. Comparative Analysis}

We have also analyzed the efficiency of different home network technologies to verify the influence of medium constraints and implementation peculiarities. In the following graphs vertical bars have been omitted for better visualization. Fig. 24 plots the efficiency for varying payload size with one sender. Ethernet and HomePNA 2.0 are the most efficient. This is expected because these protocols use collision detection. Nevertheless, the efficiency of HomePNA 3.0, which detects collisions, is similar to the efficiency of a collision-avoidance protocol. HomePNA 3.0 transmits at higher rates but, to keep compatibility with HomePNA 2.0, uses the same basic rate as HomePNA 2.0. Similarly, IEEE $802.11 \mathrm{~g}$ is less efficient than IEEE $802.11 \mathrm{~b}$ despite higher PHY rates. IEEE $802.11 \mathrm{~g}$ does not decrease the amount of time needed for overhead transmission as it does for data. HomePlug is the most efficient collision-avoidance protocol. This is due to its lower minimum contention window $\left(C W_{\text {min }}\right)$ size, which produces lower average backoff time.

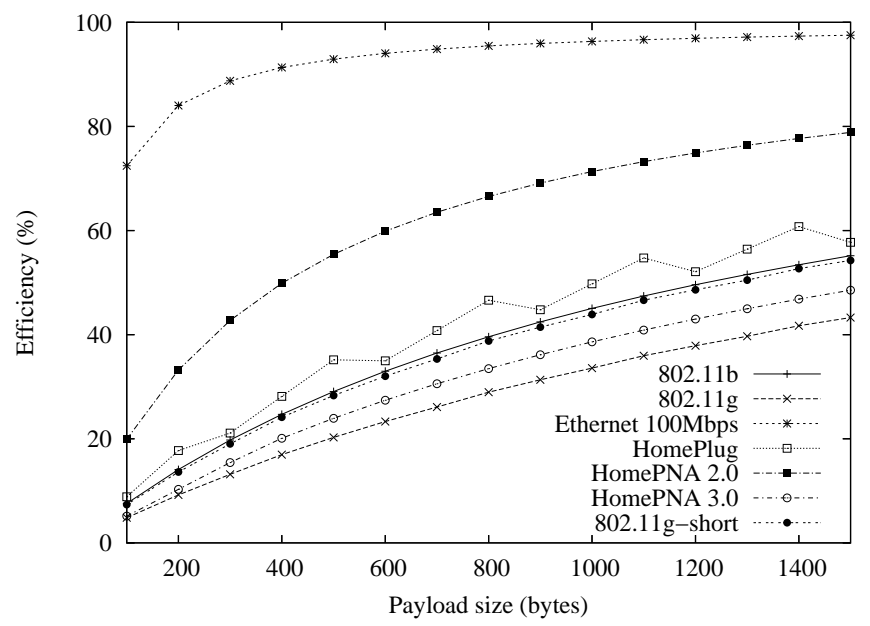

Fig. 24. Protocol efficiency for different payload sizes.

Fig. 25 plots protocol efficiency for varying number of sources. Again, collision-detection protocols react better because they can cancel transmission just after collision detection. HomePNA 3.0 has the worst performance for small number of nodes because of its low basic rate. As the number of nodes increases, its performance improves due to collision detection. HomePNA 3.0 efficiency is better than IEEE $802.11 \mathrm{~b}, 802.11 \mathrm{~g}$, and $802.11 \mathrm{~g}$-short only for 27 nodes. Moreover, $802.11 \mathrm{~g}$ is less efficient than $802.11 \mathrm{~b}$ and $802.11 \mathrm{~g}$ short due to its higher overhead. Unlike Fig. 24, where IEEE $802.11 \mathrm{~g}$ Short Slot Time presents an efficiency similar to $802.11 \mathrm{~b}$, the efficiency of $802.11 \mathrm{~g}$-short is lower than $802.11 \mathrm{~b}$ for varying number of nodes. The initial $C W_{\min }$ of IEEE $802.11 \mathrm{~g}$ Short Slot Time is lower than $802.11 \mathrm{~b}$ one, which means that initially the probability of collisions is higher for $802.11 \mathrm{~g}$-short than $802.11 \mathrm{~b}$. Similarly, HomePlug efficiency is worse than IEEE $802.11 \mathrm{~b}$ because of its lower $C W_{\min }$. As the number of nodes increases, HomePlug reacts better than IEEE $802.11 \mathrm{~b}$ because of the deferral counter.

Collision avoidance is less efficient than collision detection. Collision avoidance protocols use inter-frame spaces to guarantee that all stations are aware of current transmission. These inter-frame spaces contribute to decrease efficiency. Moreover, collision-detection protocols scale better than collisionavoidance ones due to the capacity of stopping transmissions after detecting collisions. If the collision cannot be detected, the transmitter waits for an acknowledgment and must rely on a timer expiration to conclude that the transmission has failed. Increasing the number of nodes, efficiency decrease is stronger in collision-avoidance protocols. The only exception among the protocols analyzed is HomePNA 3.0, because of backward compatibility. 


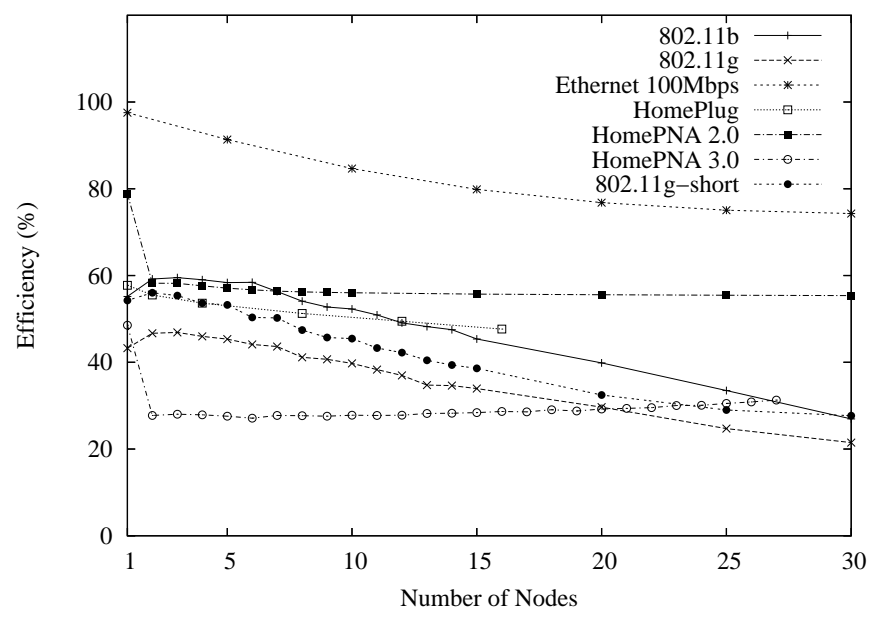

Fig. 25. Protocol efficiency for varying number of sources.

\section{CONClusion}

Currently, there is a great effort to provide communication networks to interconnect home devices. Different technologies can be classified as wired, wireless, or "no new wires". Our work has analyzed the efficiency of the most successful homenetwork technologies, emphasizing on the different access methods and MAC protocols.

First, we have derived mathematical expressions for the maximum throughput obtained in a one-node transmission by the different protocols. We have also performed similar evaluation using simulation. This analysis has shown the control overhead of each protocol for variable frame sizes. As expected, collision-detection protocols perform better than protocols that cannot detect but only avoid collisions. The exception is HomePNA 3.0, a collision-detection protocol, because it employs basic rates to keep backward compatibility. For 1500-byte frames, Ethernet, HomePNA 2.0, and HomePNA 3.0 achieve an efficiency of $97.5,78.8$, and $48.5 \%$, respectively. On the other hand, the collision-avoidance protocols HomePlug, IEEE 802.11b, and IEEE 802.11g reach 57.7, 55.2, and $43.2 \%$ efficiency, respectively. HomePNA 3.0 performs similar to collision-avoidance protocols, showing that implementation peculiarities impact the protocol efficiency. A similar unexpected result has been obtained in the IEEE 802.11 analysis. We show that IEEE $802.11 \mathrm{~b}$ is more efficient than IEEE $802.11 \mathrm{~g}$, even though IEEE $802.11 \mathrm{~g}$ achieves higher transmission rates.

Then, collision resolution mechanisms have been evaluated through simulations. We have analyzed the behavior of protocols when the stations start contending for the medium. The contention can result in collisions since we have only considered transmissions over shared mediums. Again, collisiondetection protocols perform better than the collision-avoidance ones, and once again HomePNA 3.0 is the exception. HomePNA 3.0 shows the worst efficiency for a few number of nodes due to compatibility-related constraints. Our results also show that the efficiency of collision-avoidance protocols depends on the minimum contention window $\left(C W_{\min }\right)$ size as seen with HomePlug and IEEE 802.11. Higher $C W_{\min }$ values means higher aggregate throughput when increasing number of nodes.

This work has reviewed access methods used by different shared-medium home-network protocols. Maximum throughput results obtained with mathematical analysis and simulation results have shown how efficiently each protocol shares the medium and treats collisions. Based on the analyses made, one can identify where the medium access methods may be improved and, possibly, combine the techniques used in different technologies.

\section{REFERENCES}

[1] Y.-J. Lin, H. A. Latchman, R. E. Newman, and S. Katar, "A comparative performance study of wireless and power line networks," IEEE Communications Magazine, vol. 41, no. 4, pp. 54-63, Apr. 2003.

[2] IEEE, "Carrier sense multiple access with collision detection (CSMA/CD) access method and physical layer specifications," 2005, IEEE Standard 802.3.

[3] _ - "Wireless LAN medium access control (MAC) and physical layer (PHY) specifications," 1999, IEEE Standard 802.11.

[4] _ -Wireless LAN medium access control (MAC) and physical layer (PHY) specifications: Higher-speed physical layer extension in the 2.4 GHz band," 1999, IEEE Standard 802.11b.

[5] _ "Wireless LAN medium access control (MAC) and physical layer (PHY) specifications: High-speed physical layer in the $5 \mathrm{GHz}$ band," 1999, IEEE Standard 802.11a.

[6] — "Wireless LAN medium access control (MAC) and physical layer (PHY) specifications: High-speed physical layer in the $2.4 \mathrm{GHz}$ band," 2003, IEEE Standard 802.11g.

[7] — "Part 15.1: Wireless medium access control (MAC) and physical layer (PHY) specifications for wireless personal area networks (WPANs)," 2002, IEEE Standard 802.15.1.

[8] —, "Part 15.4: Wireless medium access control (MAC) and physical layer (PHY) specifications for low-rate wireless personal area networks (WPANs)," 2006, IEEE Standard 802.15.4

[9] http://www.etsi.org, 2008

[10] http://www.homepna.org, 2008.

[11] ITU-T, "G.989.1: Phoneline networking transceivers - foundation," Feb. 2001.

[12] _ - "G.989.2: Phoneline networking transceivers - payload format and link layer requirements," Nov. 2001.

[13] _ "G.989.3: Phoneline networking transceivers - isolation function," Mar. 2003.

[14] http://www.homeplug.org, 2008.

[15] A. Dutta-Roy, "Networks for home," IEEE Spectrum, vol. 36, no. 12, pp. 26-33, Dec. 1999.

[16] J. Wang and S. Keshav, "Efficient and accurate ethernet simulation," in Proc. of the 24th Conference on Local Computer Networks (LCN'99), 1999, pp. 182-191.

[17] D. R. Boggs, J. C. Mogul, and C. A. Kent, "Measured capacity of an ethernet: Myths and reality," in Proc. SIGCOMM'88 Symposium on Communications Architectures and Protocols, 1988, pp. 222-34.

[18] M. Y. Chung, H. C. Kim, and T.-J. Lee, "HomePNA 2.0 - saturation throughput analysis," IEEE Communications Letters, vol. 7, no. 11, pp. 558-560, Nov. 2003.

[19] S. Kangude, J. Copeland, and M. Sherman, "An analysis of the Home PNA collision resolution mechanism," in 28th IEEE Conference on Local Computer Networks - LCN'2003, Bonn/Konigswinter, Germany, Oct. 2003.

[20] H. C. Kim, M. Y. Chung, T.-J. Lee, and J. Park, "Saturation throughput analysis of collision management protocol in the HomePNA 3.0 asynchronous MAC mode," IEEE Communications Letters, vol. 8, no. 7, pp. 476-478, July 2004.

[21] J. Jun, P. Peddabachagari, and M. L. Sichitiu, "Theoretical maximum throughput of IEEE 802.11 and its applications," in Proc. of the 2nd IEEE International Symposium on Network Computing and Applications (NCA'03), Cambridge, USA, Apr. 2003.

[22] Y. Xiao and J. Rosdahl, "Throughput and delay limits of IEEE 802.11," IEEE Communications Letters, vol. 6, no. 8, pp. 355-357, Aug. 2002.

[23] G. Anastasi, E. Borgia, M. Conti, and E. Gregori, "IEEE 802.11 ad hoc networks: Performance measurements," in Proc. of the 23rd International Conference on Distributed Computing Systems Workshops (ICDCSW'03), May 2003. 
[24] A. Wijesinha, Y. T. Song, M. Krishnan, V. Mathur, J. Ahn, and V. Shyamasundar, "Throughput measurement for UDP traffic in an IEEE 802.11g WLAN," in Proceedings of the Sixth International Conference on Software Engineering, Artificial Intelligence, Networking and Parallel/Distributed Computing (SNPD 05), 2005, pp. 220-225.

[25] A. Doufexi, S. Armour, B.-S. Lee, A. Nix, and D. Bull, "An evaluation of the performance of IEEE 802.11a and $802.11 \mathrm{~g}$ wireless local area networks in a corporate office environment," in Proc. of the IEEE International Conference on Communications (ICC'03), May 2003, pp. $1196-1200$.

[26] M.-H. Jung, M. Y. Chung, and T.-J. Lee, "MAC throughput analysis of HomePlug 1.0," IEEE Communications Letters, vol. 9, no. 2, pp. 184-186, Feb. 2005.

[27] M. K. Lee, R. E. Newman, H. A. Latchman, S. Katar, and L. Yonge, "Homeplug 1.0 powerline communications LANs - protocol description and performance results," International Journal of Communication Systems, vol. 16, no. 5, pp. 447-473, June 2003.

[28] E. H. Frank and J. Holloway, "Connecting the home with a phone line network chip set," IEEE Micro, vol. 20, no. 2, pp. 27-38, Apr. 2000.

[29] ITU-T, "G.PNT: PNT3 proposal overview," Aug. 2003, temporary document MC-107R1.

[30] R. Sterenson, "Guaranteed QoS in the home network environment," CopperGate Communications, Tech. Rep., 2003.

[31] ITU-T, "The proposed MAC for PNT3," Aug. 2003, temporary document PF-042.

[32] IEEE, "Media access control (MAC) bridges," 1998, IEEE Standard 802.1D.

[33] S. Gardner, B. Markwalter, and L. Yonge, "Homeplug standard brings networking to the home," Communication Systems Design Magazine, vol. 6 , no. 12 , Dec. 2000

[34] K. Dostert, Powerline Communications, $1^{\text {st }}$ ed. Prentice-Hall, 2001, ISBN 0130293423.

[35] K. Fall and K. Varadhan, The ns Manual, UC Berkeley, LBL, USC/ISI, and Xerox PARC, 2008, http://www.isi.edu/nsnam/ns/nsdocumentation.html.

[36] A. Amodei Jr., L. H. M. K. Costa, and O. C. M. B. Duarte, "Increasing the throughput of HomePNA," International Journal of Communication Systems, vol. 21, no. 7, pp. 695-711, July 2008.

[37] M. E. M. Campista, L. H. M. K. Costa, and O. C. M. B. Duarte, "Improving the Data Transmission Throughput over the Home Electrical Wiring," in $30^{\text {th }}$ IEEE Conference on Local Computer Networks (LCN'05), Sydney, Australia, Nov. 2005.

[38] P. Langfeld, "The capacity of typical powerline reference channels and strategies for system design," in International Symposium on PowerLine Communications and its Applications (ISPLC), Malmö, Sweden, Apr. 2001.

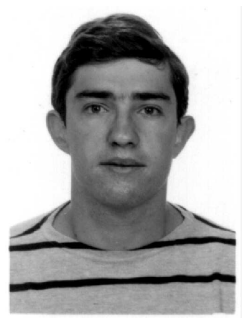

Marcelo G. Rubinstein (S'95-M'03) received a B.Sc. degree in electronics engineering and M.Sc. and D.Sc. degrees in electrical engineering from UFRJ, Brazil, in 1994, 1996, and 2001 respectively. From January to September 2000 he was at the PRiSM Laboratory, University of Versailles, France. $\mathrm{He}$ is now an associate professor with Universidade do Estado do Rio de Janeiro (UERJ). His major interests are in wireless networks, home networking, medium access control, and quality of service.

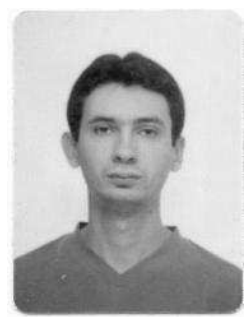

Luís Henrique M. K. Costa (M'99) received an electronic engineer degree and an M.Sc. degree in electrical engineering from the Federal University of Rio de Janeiro (UFRJ), Brazil, in 1997 and 1998, respectively, and a D.Sc. degree from the University Pierre et Marie Curie (Paris 6), Paris, France, in 2001. Since August 2004, he has been an associate professor with COPPE/UFRJ. His major research interests are in the areas of routing, wireless networks, and group communications. He has been a member of the ACM since 2001.

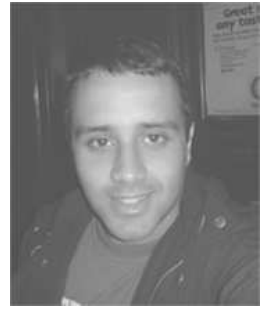

Miguel Elias M. Campista (S'05) received a telecommunications engineering degree from Fluminense Federal University (UFF), Rio de Janeiro, Brazil, in 2003 and an M.Sc. degree in electrical engineering from the Federal University of Rio de Janeiro (UFRJ), Brazil, in 2005. Currently, he is a D.Sc. student with GTA/COPPE/UFRJ. His major research interests are in multihop wireless networks, quality of service, wireless routing, and home networking.

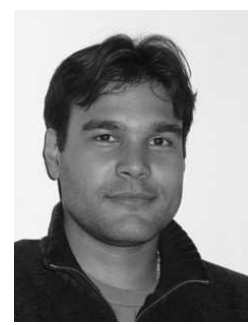

Daniel de O. Cunha received an electronic engineer degree and an M.Sc. degree in electrical engineering from Federal University of Rio de Janeiro (UFRJ), Brazil, in 2002 and 2004, respectively. In 2008, he received the Dr. degree from Federal University of Rio de Janeiro (UFRJ)/Université Pierre et Marie Curie-Paris VI. He is, currently, a post-doc researcher at the LIP6/UPMC laboratory. His major research interests are in the area of wireless networks, especially on ad hoc and sensor networks, energy conservation, and cooperative communications.

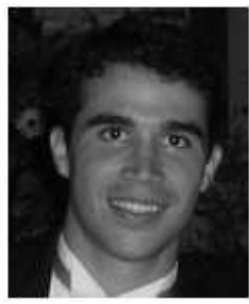

Aurelio Amodei Jr. received a telecommunications engineering degree from the Fluminense Federal University (UFF), Rio de Janeiro, Brazil, in 2003 and an M.Sc. degree in electrical engineering from the Federal University of Rio de Janeiro (UFRJ), Rio de Janeiro, Brazil, in 2005. His major research interests are in home networking, especially on IEEE 802.11 and HomePNA.

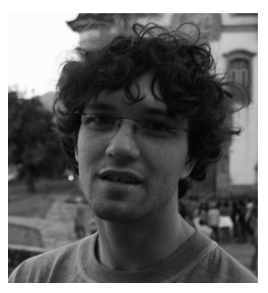

Pedro B. Velloso received an electronic engineer degree and an M.Sc. degree in electrical engineering from the Universidade Federal do Rio de Janeiro in 2001 and 2003, respectively. He received the $\mathrm{PhD}$ degreee from the Université Pierre et Marie Curie (Paris 6) in 2008. Currently, he is post-doc researcher at Laboratoire d'Informatique de Paris 6. His interests are in distributed multimedia applications, wireless communications, home networks, and security.

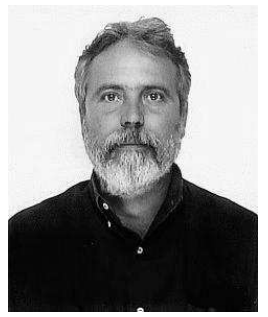

Otto Carlos M. B. Duarte received electronic engineer and M.Sc. degrees from UFRJ, Rio de Janeiro, Brazil, in 1976 and 1981, respectively, and a Dr.Ing. degree from ENST/Paris, France, in 1985 Since 1978 he has been a professor with UFRJ. From January 1992 to June 1993 he was with MASI Laboratory, Université Paris 6, Paris. In 1995 he spent three months with the International Computer Science Institute (ICSI), University of California, Berkeley. In 1999, 2001, and 2006 he was an invited professor at Université Paris 6. His major research interests are in multicast, QoS guarantees, security, and mobile communications. 